\title{
Experimentelle Untersuchungen über die blutzuckersenkende Wirkung von Rinder-Proinsulin
}

\author{
W. Puls u. G. KRONeBerg
}

Institut für Pharmakologie der Farbenfabriken Bayer AG, Werk Elberfeld

Eingegangen am 10. Dezember 1968

\begin{abstract}
Experimental investigations of the hypoglycaemic action of bovine proinsulin

Summary. Proinsulin obtained from bovine insulin (Schmod and AREns [6]) lowered the blood sugar level of rats and mice. The activity ratio proinsulin : insulin was $1: 4$ in fod rats and fasted mice, and $1: 2$ in fasted rats, based on an equimolar dosage and measuring the effect in terms of maximum lowering of the blood sugar value. After proinsulin the decrease of the blood sugar was more protracted than after insulin. Proinsulin which was incubated with trypsin in vitro, resembled insulin in degree and onset of action. In pancreatectomized, nephrectomized and two-thirds hepatectomized rats proinsulin acted as in normal animals. Alloxan-diabetic mice responded to proinsulin like intact mice. The reason why the onset of the hypoglycaemic action of proinsulin was delayed is probably due to the fact that the active insulin must first be produced by a time-dependent proteolytic process. This may also be the cause of the ineffectiveness of proinsulin in the mouse convulsion test which was quoted by RUBENsTwIN et al. [5].
\end{abstract}

Etude expérimentale de l'action hypoglycémiante de la pro-insuline de boeuf

Résumé. Chez les rats et les souris le taux du sucre sanguin est abaissé par une proinsuline obtenue à partir de l'insuline bovine (Sohmidt et Arens [6]). Chez les rats alimentés et les souris à jeun, le rapport d'activité proinsuline: insuline est de $1: 4$, chez les rats à jeun de $1: 2$, dans le cas de dose équimolaire et en mesurant l'abaissement maximum du sucre sanguin. L'abaissement du sucre sanguin évolue de façon plus lente après proinsuline qu'après insuline. La proinsuline, incubée in vitro avec la trypsine, agit quantitativement et au point de vue temps, comme l'insuline. Chez les rats pancréatectomisés, chez les rats néphrectomisés ainsi quo chez coux hépatectomisés aux dewx tiers, l'action de la proinsuline est la même que chez les animaux normaux. Les souris rendues diabétiques par l'alloxane répondent également à la proinsuline comme les souris intactes. L'action hypoglycémiante de la proinsuline se manifeste de façon retardée probablement parce que l'insuline active doit tout d'abord se former par un processus protéolytique dépendant du facteur temps. Ceci pourrait être aussi la raison pour laquelle, RuBEnsters et coll. [5] signalent l'absence d'effet de la proinsuline dans le test de convulsion chez la souris.

Zusammenfassung. An Ratten und Mäusen wird der Blutzucker durch ein aus Rinderinsulin gewonnenes Proinsulin (SCHMIDT und ARENS [6]) gesenkt. Bei gefütterten Ratten und nüchternen Mäusen ist das Wirkungsverhältnis Proinsulin: Insulin $=1: 4$, bei nüchternen Ratten $1: 2$, wenn äquimolar dosiert und das Maximum der Blutzuckersenkung gewertet wird. Die Blutzuckersenkung verläuft nach Proinsulin protrahierter als nach Insulin. Proinsulin, welches in vitro mit Trypsin inkubiert wurde, wirkt quantitativ und zeitlich wie Insulin. Bei pankreatektomierten, bei nephrektomierten und bei zweidrittelhepatektomierten Ratten wirkt Proinsulin ebenso wie an Normaltieren. Auch alloxandiabetische Mäuse sprechen auf Proinsulin wie intakte Mäuse an. Die hypoglykämische Wirkung des Proinsulins tritt wahrscheinlich deshalb verzögert ein, weil das aktive Insulin in einem zeitabhängigen proteolytischen Prozeß erst entstehen muß. Dies könnte auch die Ursache für eine von RuBEnstein et al. [5] zitierte, fehlende Wirkung von Proinsulin im Mäusekrampftest sein.

Key-words: Insulin, proinsulin, blood sugar, mice, rats, pancreatectomy, nephrectomy, hepatectomy (partial) alloxan diabetes.
STEINER et al. konnten vor kurzem einen einkettigen Vorläufer des Insulins, das Proinsulin, bei der Inkubation von Insulinomgewebe [8] und isolierten $\beta$-Zellen aus Rattenpankreas [9] nachweisen. Nach Untersuchungen von SCHMid T und ARexs [6] ist im Proinsulin vom Rind das Aminoende der Insulin-AKette mit dem Carboxylende der Insulin-B-Kette durch ein aus 29 Aminosäuren bestehendes Verbindungsglied verknüpft. In in vitro-Untersuchungen an isolierten Fettzellen und am Froschmuskel zeigte Proinsulin nur eine sehr geringe Insulinwirkung (zit. bei Rubenstern et al. [5]). Die Inkubation von Proinsulin in vitro mit Trypsin ergibt Des-Ala-Insulin als Umwandlungsprodukt, das sich elektrophoretisch [6] und biologisch im Mauskrampftest [3] nicht vom Insulin unterscheidet. Über die Umwandlung von Proinsulin in vivo ist noch nichts bekannt. Auch Untersuchungen über die Blutzuckerwirkung sind bisher nicht durch- geführt worden. Wir hatten Gelegenheit, das von Schmid und Aress gewonnene Proinsulin auf seine Blutzuckerwirkung an Ratten und Mäusen zu untersuchen und möchten im Folgenden darüber berichten.

\section{Material und Methoden}

Tiermaterial: Es wurden Ratten beiderlei Geschlechts vom Stamm Wistar/Winkelmann-SPF und weibliche Mäuse des Stammes NMRI/Hann.-SPF verwendet. Sie erhielten Leitungswasser und Standardfutter Altomin-R ad libitum. "Gefütterten" Ratten wurde das Futter unmittelbar, „nüchternen“ Ratten und Mäusen $16 \mathrm{Std}$ vor Versuchsbeginn entzogen. Die Stalltemperatur betrug. $24 \pm 1^{\circ} \mathrm{C}$, die Beleuchtungszeit (hell/dunkel) 12/12 Std. Das Körpergewicht der intakten weiblichen Ratten betrug $110-130 \mathrm{~g}$, der pankreatektomierten männlichen Versuchstiere $175-195 \mathrm{~g}$, der hepatektomierten männlichen sowie nephrektomierten weiblichen Ratten $130-160 \mathrm{~g}$ bzw. $120-145 \mathrm{~g}$. 
Substanzen: Als Standard wurde ein Mischinsulin von Rind und Schwein mit 24 IE/mg (int. Standard IV 1958) verwendet. Proinsulin stellte Dr. D.D. ScнmmotBiochemisches Laboratorium der Farbenfabriken Bayer AG, Werk Elberfeld, zur Verfügung, wofür wir ihm herzlich danken. Insulin und Proinsulin wurden in PhosphatZitrat-Puffer pH 8 gelöst. Das Injektionsvolumen betrug $2 \mathrm{ml} / \mathrm{kg}$ Ratte bzw. $10 \mathrm{ml} / \mathrm{kg}$ Maus. Trasylol ${ }^{\circledR}$ lag als Trockensubstanz mit $4900 \mathrm{KIE} / \mathrm{mg}$ vor. Es wurde in physiologischer Kochsalzlösung gelöst. Das Injektions. volumen betrug $10 \mathrm{ml} / \mathrm{kg}$ Ratte. In den Versuchen mit Blutentnahmen nach 10,20 und 30 Min. (Abb. 1 und Tabelle 7) wurde zu jedem Zeitpunkt ein anderes Tierkollektiv benutzt. Die übrigen Versuche wurden an jeweils demselben Tierkollektiv durchgeführt. Dieses unterschiedliche Vorgehen war notwendig, weil die Höhe des Blutzuckers durch so schnell aufeinanderfolgende Punktionen, wie es bei den 10-Minutenabständen der Fall ist, bereits verändert werden kann.

Methoden: Blutzuckerbestimmungen wurden mit dem Auto-Analyzer/Technicon nach der von HoFrMAN [4] angegebenen Kaliumferricyanid-Methode durchgeführt. Das Blut wurde dem retroorbitalen Venenplexus entnommen. - Die Pankreatektomie erfolgte in Evipan ${ }^{\circledR}$-Narkose (100 mg/kg i.p.) nach Cox et al. [2] durch Ligatur von Pankreas, angrenzender Duodenalschleife und Milz. Leberarterie und Pfortader blieben intakt. - Die Zweidrittelhepatektomie wurde in Äthernarkose vorgenom. men. Die beiden großen ventralen Leberlappen wurden nacheinander ligiert und reseziert. Kontrollversuche an 10 Ratten ergaben, daß bei diesem Vorgehen $69 \pm 2.0 \%$ bzw. 26 Prozent, bezogen auf die Kontrollgruppe. Nach $20 \mathrm{Min}$. ist die Blutglucose stärker, nämlich um 15 bzw. 41 Prozent (Abb. 1B) and nach 30 Min. um 24 bzw. 44 Prozent (Abb. 1C) vermindert. Demgegenüber führen annähernd äquimolare Dosen Proinsulin ${ }^{1}$ (0.8 bzw. $2.0 \mathrm{nmol} / \mathrm{kg} \mathrm{s.c.)}$ während der ersten $30 \mathrm{Min}$. nach der Applikation zu keiner signifikanten Veränderung des Blutzuckers. Erst nach einer Stunde besteht eine signifikante Differenz zur Kontrolle. Auch die etwa fünffach höhere Proinsulindosis, nämlich $4.1 \mathrm{nmol} /$ $\mathrm{kg}$ s. c., ist nach $10 \mathrm{Min}$. noch unwirksam. Nach $20 \mathrm{Min}$., ein Zeitpunkt, zu dem Insulin in der halben äquimolaren Menge den Blutzucker um 41\% senkt, ist die Wirkung des Proinsulins nur eben so stark wie die Wirkung von $0.7 \mathrm{nmol} / \mathrm{kg}$ Insulin. Erst nach 30 und $60 \mathrm{Min}$. ist der hypoglykämische Effekt dieser Proinsulindosis ausgeprägt, allerdings nicht stärker als der mit der halben Insulindosis erreichte. Proinsulin wirkt demnach verzögert und quantitativ schwächer als Insulin.

\section{Gefütterte Ratten}

Erwartungsgemäß wirkt Insulin, wie ein Vergleich der Werte in Tabelle 1 mit denen der Abb. 1 zeigt, an gefütterten Ratten deutlich schwächer als an nüchter-

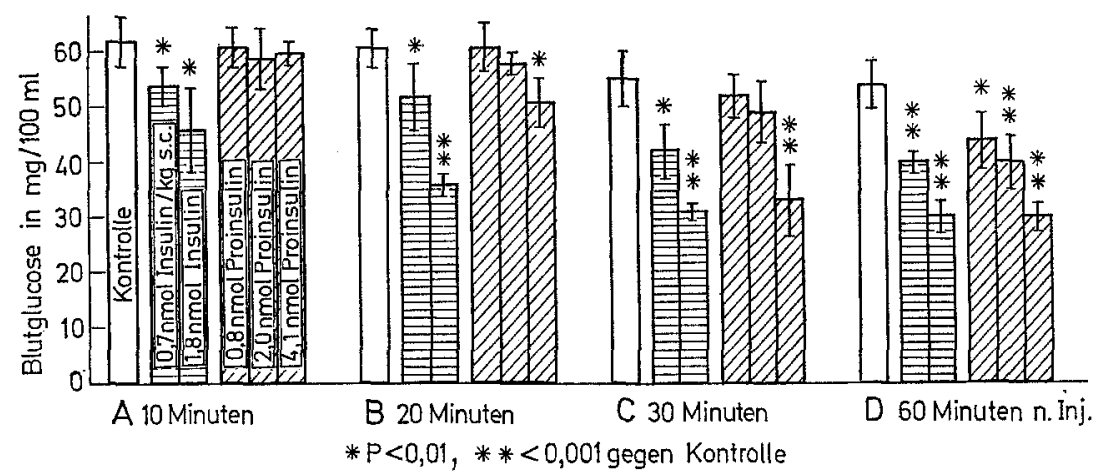

Abb. 1. Blutzuokerwerte in $\mathrm{mg} / 100 \mathrm{ml}+1 \mathrm{~s}$ von nüchternen Ratten zu verschiedenen Zeiten nach subcutaner Injektion verschiedener Dosen Insulin bzw. Proinsulin (nmol/kg): $\mathbf{n}=6$. Zu jedem Zeitpunkt der Blutzuckeruntersuchung wurden jeweils andere Tierkollektive verwendet (s. Methode)

der Leber entfernt werden. - An weiblichen Ratten erfolgte die Nephrektomie in üblicher Weise durch Ligieren der zu- und abführenden Gefäße und Entfernen der Nie. ren. Alle Operationen wurden unmittelbar vor Versuchsbeginn ausgeführt. - Zur Erzeugung eines Alloxandiabetes erhielten Mäuse $100 \mathrm{mg} / \mathrm{kg}$ Alloxantetrahydrat $/ 10 \mathrm{ml}$ Zitratpuffer $\mathrm{pH} 5$ in 30 sec i.v. injiziert. Es wurden nur solche Tiere verwendet, bei denen 4 Tage später eine Nüchternghucosurie (Glukotest (1-Streifen) nachweisbar war. - Signifikanzberechnungen erfolgten nach STUDENT.

\section{Ergebnisse}

\section{Nüchterne Ratten}

Wie die Abb, $1 \mathrm{~A}$ zeigt, senken 0.7 und $1.8 \mathrm{nmol}$ Insulin den Blutzucker nüchterner Ratten schon 10 Min. nach subcutaner Injektion signifikant um 13 nen. Auch der Effekt von Proinsulin ist reduziert: $4.1 \mathrm{nmol} / \mathrm{kg}$, die in den Versuchen an nüchternen Tieren stark wirksame Dosis, hat noch keine Wirkung. Das gilt selbst für die doppelte Dosis von $8.2 \mathrm{nmol} / \mathrm{kg}$ (Tabelle 1). Erst $16.5 \mathrm{nmol} / \mathrm{kg}$ Proinsulin haben eine mit $3.7 \mathrm{nmol} / \mathrm{kg}$ Insulin etwa vergleichbare Wirksamkeit. Auf molarer Basis entspricht dies einem Wirksamkeitsverhältnis von ca. 1:4.

1 Das aus der Aminosäurenanalyse berechnete Molekulargewicht unseres Proinsulins beträgt nach SCHMIDT u. ArENS [6] 8621, das Molekulargewicht von Rinderinsulin 5733. In unseren Untersuchungen sind nur annähernd äquimolare Dosen von Insulin und Proinsulin benutzt, da für unser Proinsulin ursprünglich ein höheres Molekular. gewicht, nämlich 9500, angenommen wurde. 


\section{Pankreatektomierte Ratten}

Der Blutzucker von gefütterten Ratten steigt nach Ausschaltung des Pankreas kontinuierlich an (Tabelle 2). Durch $7.3 \mathrm{nmol}$ Insulin $/ \mathrm{kg}$ s. c. läßt sich der Anstieg signifikant hemmen. Eine annähernd äquimolare Dosis Proinsulin, $8.2 \mathrm{nmol}$, ist wirksam, aber deutlich schwächer. Die vierfache Dosis Proinsulin führt zu einer

\section{Zweidrittelhepatektomierte Ratten}

Die Injektion von $5.5 \mathrm{nmol}$ Insulin/kg s. c. führt bei zweidrittelhepatektomierten, gefütterten Ratten zu einer signifikanten Verminderung der Blutglucose (Tabelle 3$)$. Eine äquimolare Dosis Proinsulin $(6.2 \mathrm{nmol} / \mathrm{kg}$ s.c.) senkt den Blutzucker nicht signifikant, die vierfache Menge hingegen etwa ebenso stark wie $5.5 \mathrm{nmol}$

Tabelle 1. Blutzuckersenkende Wirkung von Insulin und Proinsulin an gefütterten Ratten $z u$ verschiedenen Zeiter rach subcutaner Applikation; $n=6$

\begin{tabular}{|c|c|c|c|c|}
\hline \multirow[b]{2}{*}{ Dosis/kg s.c. } & \multicolumn{4}{|c|}{ Blutglucose in $\mathrm{mg} / 100 \mathrm{ml} \pm \mathrm{ls}$} \\
\hline & $\overline{0}$ & 30 & 60 & 90 Min. n. Appl. \\
\hline Kontrolle & $101 \pm 5.5$ & $112 \pm 8.0$ & $118 \pm 7.8$ & $117 \pm 8.3$ \\
\hline $3.7 \mathrm{nmol}$ Insulin & $96 \pm 3.8$ & $\underline{\underline{79 \pm 7.5}}$ & $\underline{101 \pm 12}$ & $\underline{107 \pm 5.8}$ \\
\hline $7.3 \mathrm{nmol}$ Insulin & $101 \pm 2.3$ & $61 \pm 4.7$ & $68 \pm 8.4$ & $89 \pm 15$ \\
\hline $4.1 \mathrm{nmol}$ Proinsulin & $102 \pm 2.3$ & $112 \pm 3.4$ & $118 \pm 2.1$ & $\overline{124 \pm 13}$ \\
\hline 8.2 nmol Proinsulin & $103 \pm 3.5$ & $102 \pm 3.9$ & $112 \pm 3.0$ & $112 \pm 3.4$ \\
\hline 16.5 nmol Proinsulin & $99 \pm 8.1$ & $79 \pm 10$ & $86 \pm 13$ & $101 \pm 16$ \\
\hline
\end{tabular}

$\left.\begin{array}{l}=P<0.05 \\ =P<0.001\end{array}\right\}$ gegen Kontrolle

Tabelle 2. Blutzuckersenkende Wirkung von Insulin und Proinsulin an gefütterten pankreatektomierten Ratten. Der Nullwert wurde unmittelbar nach der Operation bestimmt. $n=4$, Kontrolle $n=6$

\begin{tabular}{|c|c|c|c|c|c|}
\hline \multirow[b]{2}{*}{ Dosis/kg s.c. } & \multicolumn{5}{|c|}{ Blutglucose in $\mathrm{mg} / 100 \mathrm{ml} \pm \mathrm{ls}$} \\
\hline & 0 & 30 & 60 & 90 & 120 Min. n. Appl. \\
\hline Kontrolle & $114 \pm 11$ & $157 \pm 17$ & $178 \pm 29$ & $225 \pm 41$ & $253 \pm 49$ \\
\hline 7.3 nmol Insulin & $115 \pm 14$ & $\underline{79 \pm 13}$ & $95 \pm 34$ & $\underline{128} \pm 58$ & $143 \pm 50$ \\
\hline 8.2 nmol Proinsulin & $116 \pm 16$ & $125 \pm 12$ & $138 \pm 13$ & $175 \pm 8.0$ & $179 \pm 45$ \\
\hline $33.0 \mathrm{nmol}$ Proinsulin & $112 \pm 4.0$ & $61 \pm 13$ & $49 \pm \quad 9.2$ & $58 \pm \quad 5.3$ & $70 \pm \quad 3.5$ \\
\hline
\end{tabular}

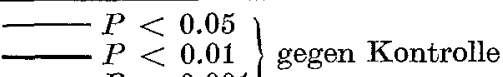

$=P<0.001$

Tabelle 3. Blutzuckersenkende Wirkung von Insulin und Proinsulin an gefütterten zweidrittelhopatektomierten Ratten zu verschiedenen Zeiten nach subcutaner Applikation. Der Nullwert wurde unmittelbar nach der Operation bestimmt. $n=6$

\begin{tabular}{|c|c|c|c|c|c|}
\hline \multirow[b]{2}{*}{ Dosis/kg s.c. } & \multicolumn{5}{|c|}{ Blutglucose in $\mathrm{mg} / 100 \mathrm{ml} \pm \mathrm{ls}$} \\
\hline & 0 & 30 & 60 & 90 & 120 Min. n. Appl. \\
\hline Kontrolle & $106 \pm 8.6$ & $121 \pm 16$ & $118 \pm 19$ & $119 \pm 15$ & $119 \pm 12$ \\
\hline $5.5 \mathrm{nmol}$ Insulin & $108 \pm 5.2$ & $62 \pm 11$ & $\underline{62 \pm 11}$ & $67 \pm 12$ & $\underline{73 \pm 19}$ \\
\hline $6.2 \mathrm{nmol}$ Proinsulin & $110 \pm 4.7$ & $110 \pm 8.1$ & $107 \pm 14$ & $115 \pm 15$ & $116 \pm 17$ \\
\hline 24.7 nmol Proinsulin & $107 \pm 4.4$ & $70 \pm 10$ & $60 \pm 7.2$ & $61 \pm 7.7$ & $61 \pm 11$ \\
\hline
\end{tabular}

$=P<0.001$ gegen Kontrolle

stärkeren und länger andauernden Hypoglykämie als 7.3 nmol Insulin. Proinsulin wirkt demnach auch an pankreaslosen Ratten, und zwar eher stärker und anhaltender als an intakten. Für eine Wirkung des Proinsulins in vivo, möglicherweise als Aktivierungsorgan, ist das Pankreas also nicht erforderlich.
Insulin. Das Wirkungsverhältnis Insulin:Proinsulin ist wiederum 1:4 wie bei intakten Tieren. Für die Wirkung des Proinsulins hat die Leber offensichtlich keine entscheidende Bedeutung, wenn auch nicht ausgeschlossen werden kann, daß der verbliebene Leberrest genügt, um das applizierte 
Proinsulin zur wirksamen Insulinform umzuwandeln.

\section{Nephrektomierte Ratten}

An nephrektomierten Ratten wurde der Blutzucker durch $4.1 \mathrm{nmol} / \mathrm{kg}$ Proinsulin (Tabelle 4) ebenfalls stark und verzögert gesenkt. Vergleicht man die Werte der Tabelle 4 mit den als „Kontrollen" anzusehenden licherweise nur partiell, so daß eine ausreichende Proteinasen-Restaktivität noch vorhanden ist.

\section{Intakte nüchterne Mäuse}

Bei Mäusen steigt der Blutzucker an sich schon während des Versuchsablaufs an (Kontrolle in Tabelle 5). Es dürfte sich um die Folge der in kurzen Intervallen vorgenommenen Venenpunktionen im Orbital-

Tabelle 4. Blutzuckersenkende Wirkung von Proinsulin an nephrektomierten nüchternen Ratten zu verschiedenen Zeiten nach subcutaner Applikation

Proinsulin wurde 1 Std nach der Operation s.c., Trasylol unmittelbar nach der Operation i.p. injiziert. Der Nullwert wurde 1 Std nach der Operation bestimmt. $n=5$

\begin{tabular}{|c|c|c|c|c|c|}
\hline \multirow[b]{2}{*}{ Dosis/kg } & \multicolumn{5}{|c|}{ Blutglucose in $\mathrm{mg} / 100 \mathrm{ml} \pm \mathrm{ls}$} \\
\hline & 0 & 30 & 60 & 90 & 120 Min. n. Appl. \\
\hline Kontrolle & $70 \pm 12$ & $66 \pm 9.5$ & $59 \pm$ & $59 \pm 10$ & $60 \pm 9.4$ \\
\hline 4.1 nmol Proinsulin s.c. & $69 \pm 6.8$ & $53 \pm 6.8$ & $33 \pm \quad 2.9$ & $32 \pm 3.4$ & $35 \pm 4.7$ \\
\hline $\begin{array}{l}4.1 \text { nmol Proinsulin s.c. }+ \\
200000 \text { KIE Trasylol i.p. }\end{array}$ & $76 \pm 5.1$ & $56 \pm 9.7$ & $34 \pm \quad 17$ & $41 \pm 16$ & $42 \pm 16$ \\
\hline
\end{tabular}

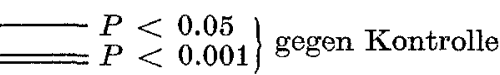

Tabelle 5. Blutzuckersenkende Wirkung von Insulin und Proinsulin an intakten nüchternen Mäusen zu verschiedenen Zeiten nach subcutaner Applikation. $n=7$

\begin{tabular}{|c|c|c|c|c|}
\hline \multirow[b]{2}{*}{ Dosis/kg s.c. } & \multicolumn{4}{|c|}{ Blutglucose in $\mathrm{mg} / 100 \mathrm{ml} \pm \mathrm{ls}$} \\
\hline & 0 & 30 & 60 & 90 Min. n. Appl. \\
\hline Kontrolle & $68 \pm 10$ & $112 \pm 20$ & $135 \pm 23$ & $164 \pm 24$ \\
\hline $1.8 \mathrm{nmol}$ Insulin & $64 \pm 7.5$ & $76 \pm 13$ & $89 \pm 29$ & $119 \pm 37$ \\
\hline $3.7 \mathrm{nmol}$ Insulin & $71 \pm 8.4$ & $63 \pm 11$ & $48 \pm 13$ & $69 \pm 26$ \\
\hline $7.3 \mathrm{nmol}$ Insulin & $65 \pm 11$ & $\overline{34 \pm 2.6}$ & $\overline{30 \pm 6.4}$ & $37 \pm 8.5$ \\
\hline $4.1 \mathrm{nmol}$ Proinsulin & $70 \pm 7.5$ & $93 \pm 16$ & $113 \pm 24$ & $136 \pm 26$ \\
\hline 8.2 nmol Proinsulin & $78 \pm 9.2$ & $94 \pm 40$ & $\underline{99 \pm 30}$ & $111 \pm 45$ \\
\hline 16.5 nmol Proinsulin & $72 \pm 14$ & $61 \pm 21$ & $53 \pm 5.8$ & $57 \pm 13$ \\
\hline
\end{tabular}

der Abb. 1, so könnte Proinsulin an nephrektomierten Tieren eher wirksamer sein als bei den intakten. Die Niere ist also für die Umwandlung des Proinsulins zum aktiven Produkt nicht verantwortlich. Durch sie geht Proinsulin und Insulin, wie RuBensteres et al. [5] zeigten, im Harn verloren.

$\mathrm{Zu}$ der Frage, ob Trasylol-hemmbare Proteinasen an der Umformung von Proinsulin beteiligt sind, haben wir Trasylol und Proinsulin kombiniert verabfolgt: zunächst erhielten die Tiere $200000 \mathrm{KIE}$ Trasylol/kg i.p., 60 Min. später das Proinsulin s.c. Wie die Werte der Tabelle 4 demonstrieren, bleibt die Proinsulinwirkung unter Trasylol unverändert. Dieser ,negative " Befund berechtigt allerdings nicht zu der Schlußfolgerung, daß Proteinasen an der Proinsulinumwandlung nicht beteiligt sind. Die Hemmwirkung des Trasylol ist mög- plexus handeln. - Subcutane Injektionen von 1.8, 3.7 bzw. $7.3 \mathrm{nmol} / \mathrm{kg}$ Insulin s.c. senken den Blutzucker dosisabhängig (Tabelle 5). Die kleinste applizierte Dosis Proinsulin, $4.1 \mathrm{nmol} / \mathrm{kg}$, beeinflußt die Blutglucose nicht signifikant. Die Erhöhung der Dosis auf $8.2 \mathrm{bzw} .16 .5 \mathrm{nmol} / \mathrm{kg}$ Proinsulin führt zu einer dosisabhängigen Verminderung der Glucose im Blut. 16.5 $\mathrm{nmol} / \mathrm{kg}$ Proinsulin sind ungefähr ebenso wirksam wie $3.7 \mathrm{nmol} / \mathrm{kg}$ Insulin. - Das Wirkungsverhältnis Insulin:Proinsulin ist 1:4.

\section{Alloxandiabetische nüchterne Mäuse}

Der erhöhte Blutzucker alloxandiabetischer Mäuse wird durch $5.5 \mathrm{nmol} / \mathrm{kg}$ Insulin s.c. innerhalb einer Stunde sehr stark vermindert (Tabelle 6). Proinsulin führte in einer Dosierung von $12.4 \mathrm{nmol} / \mathrm{kg}$ s.c. eben- 
falls zu einer signifikanten, aber nicht so ausgeprägten Hypoglykämie. Die doppelte Dosis Proinsulin, nämlich $24.7 \mathrm{nmol}$, sind eine Stunde nach ihrer Injektion nicht stärker wirksam. Alloxandiabetische Mäuse sind sowohl gegen Insulin als auch Proinsulin sehr empfindlich. Auffällig ist die größere Streuung der Proinsulinwerte (s. Tab. 6, 60-Min.-Werte).

Tabelle 6. Blutzuckersenkende Wirkung von Insulin und Proinsulin an alloxandiabetischen nüchternen Mäusen nach subcutaner Applikation. $n=6$

\begin{tabular}{llc}
\hline & \multicolumn{2}{l}{ Blutglucose in $\mathrm{mg} / 100 \mathrm{ml} \pm \mathrm{ls}$} \\
\cline { 2 - 3 } Dosis/kg s.c. & 0 & $60 \mathrm{Min} . \mathrm{n}$. Appl. \\
Kontrolle & $450 \pm 45$ & $380 \pm 59$ \\
$5.5 \mathrm{nmol}$ Insulin & $410 \pm 30$ & $\frac{66 \pm 22}{\overline{37 \pm 123}}$ \\
$12.4 \mathrm{nmol}$ Proinsulin & $418 \pm 80$ & $\frac{130.98}{171 \pm 0}$ \\
24.7 nmol Proinsulin & $504 \pm 36$ & $\underline{171 \pm 0}$ \\
\hline
\end{tabular}

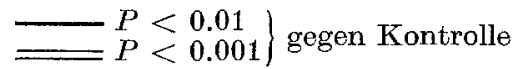

\section{Diskussion}

Sreiner et al. [9] zeigten, daß die Biosynthese des Insulins über Proinsulin führt. Proinsulin kann aber nur in geringen Mengen aus kristallinem Insulin $[6,7,1]$ gewonnen werden. Das Pankreas enthält und sezerniert offenbar überwiegend biologisch aktives Insulin und relativ wenig Proinsulin. Dementsprechend ist zu folgern, daß die Herausspaltung des aus 29 solches sowohl an isolierten Fettzellen als auch am isolierten Froschmuskel praktisch keine insulinartige Wirkung hat.

Gibt man Proinsulin intakten Tieren, so erhält man, wie wir zeigen konnten, eindeutige Senkungen des Blutzuckers. Der Unterschied zum Insulin liegt in einer zeitlichen Verschiebung des Wirkungseintritts und einer geringeren, das Maximum betreffenden Wir. kungsintensität. Beides ist aus der Vorstellung heraus verständlich, daß Proinsulin innerhalb einer gewissen Zeit erst zu Insulin umgewandelt werden muß; denn infolge der konstanten Inaktivierungsbedingungen wird bei langsamerem ,Anfluten" aktiven Materials nie die gleich hohe Maximalkonzentration wie bei Gabe eines von vornherein fertigen Insulins erreicht. Für die Richtigkeit dieser Vorstellung sprechen vor allem Befunde über die Wirkung von in vitro aktiviertem Proinsulin, die in Tabelle 7 zusammengefaßt sind: während Proinsulin erwartungsgemäß mit deutlicher Latenz wirksam wird, verhält es sich nach vorheriger in vitroInkubation mit Trypsin genau wie die äquimolare Menge reinen Insulins. (Wegen Substanzmangel konnten die Versuche nur mit einer Dosis durchgeführt werden.) Da sich Proinsulin also durch Trypsin vollständig zu aktivem Insulin (Des-Ala-Insulin!) umwandeln läßt, wird infolgedessen auch der Einwand entkräftet, daß die im Vergleich mit Insulin schwächere hypoglykämische Proinsulinwirkung die Folge einer partiellen Denaturierung des benutzten Proinsulins sei. - An welchem Ort injiziertes Proinsulin zu Insulin aktiviert

Tabelle 7. Blutzuckersenkende Wirkung von Insulin, mit Trypsin inkubiertem Proinsulin bzw. Proinsulin an nüchternen Ratten zu verschiedenen Zeiten nach subcutaner Applikation

Proinsulin wurde $22 \mathrm{Min}$. lang bei $37^{\circ} \mathrm{C}$ in Trispuffer $\mathrm{pH} 8$ inkubiert. $n=6 . \mathrm{Zu}$ jedem Zeitpunkt der Blutzuckeruntersuchung wurden jeweils andere Tierkollektive verwendet (s. Methode)

\begin{tabular}{|c|c|c|c|}
\hline \multirow[b]{2}{*}{ Dosis/kg s.c. } & \multicolumn{3}{|c|}{ Blutglucose in $\mathrm{mg} / 100 \mathrm{ml} \pm$ ls } \\
\hline & 10 & 20 & 30 Min. n. Appl. \\
\hline Kontrolle & $66 \pm 4.7$ & $66 \pm 5.7$ & $63 \pm 4.4$ \\
\hline $1.8 \mathrm{nmol}$ Insulin & $60 \pm 7.2$ & $40 \pm 1.8^{\mathrm{b}}$ & $38 \pm 2.2^{\mathrm{a}}$ \\
\hline $\begin{array}{l}2.0 \text { nmol mit Trypsin } \\
\text { inkub. Proinsulin }\end{array}$ & $58 \pm 4.4$ & $40 \pm 2.0^{\mathrm{b}}$ & $\underline{39 \pm 5.7^{\mathrm{a}}}$ \\
\hline 2.0 nmol Proinsulin & $65 \pm 8.3$ & $60 \pm 2.6$ & $46 \pm 5.3$ \\
\hline
\end{tabular}

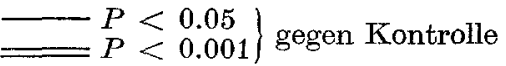

$$
\begin{aligned}
& \left.\begin{array}{l}
\text { a } P<0.05 \\
\text { b } P<0.001
\end{array}\right\} \text { gegen Proinsulin-Gruppe }
\end{aligned}
$$

Aminosäuren bestehenden und die beiden Insulinketten verbindenden Peptids in den $\beta$-Zellen erfolgen muß. Nach STEINER (pers. Mitteilung) ist es in hohem Grade unwahrscheinlich, daß Insulin im pharmazeutischen Herstellungsprozeß aus Proinsulin entsteht.

Für die bereits im Pankreas stattfindende Umwandlung von Proinsulin sprechen auch die von Rubenstein [5] zitierten Befunde, daß Proinsulin als wird, ließ sich in unseren Versuchen nicht ermitteln. Da Proinsulin auch an pankreatektomierten und alloxandiabetischen Tieren im gleichen quantitativen Verhältnis zu Insulin wie an Normaltieren wirksam war,kann es offenbar auch extrapankreatisch voll aktiviert werden. Ebenso unabhängig scheint die Umwandlung auch von der Niere zu sein, da Proinsulin an nephrektomier. ten Tieren die volle Wirkung hatte. Sie war eher stärker 
als bei intakten Kontrollen, offensichtlich deshalb, weil Insulin und Proinsulin durch die Niere in den Harn eliminiert werden [5]. Mit Sicherheit läßt sich die Niere als partieller Umwandlungsort allerdings nicht ausschließen, da die beim nephrektomierten Tier weniger gebildete Insulinmenge durch fehlende Ausscheidung des an anderen Orten entstehenden Insulins kompensiert werden kann.

Leider haben die Versuche an zweidrittelhepatektomierten Ratten kein verwendbares Ergebnis gebracht, da die Proinsulinwirkung auch bei diesen Tieren unbeeinträchtigt war, obwohl anzunehmen ist, daß die Leber zur Umwandlung von Proinsulin befähigt ist. Offensichtlich reicht dazu aber ein Drittel des Organs aus. Die Frage müßte unter anderen experimentellen Bedingungen untersucht werden.

Unsere Ergebnisse stehen in scheinbarem Widerspruch zu Äußerungen von RuBEnsteIs et al. [5]. Die Autoren zitieren eine persönliche Mitteilung, nach der Proinsulin im Mäusekrampftest eine ,extrem niedrige biologische Wirksamkeit" hat. Da wir die methodischen Bedingungen der betreffenden Untersuchungen nicht kennen, läßt sich die Diskrepanz schwer beurteilen. Wir möchten aber annehmen, daß deren wesentliche Ursache in der relativ langsamen Entstehung des aktiven Insulins liegt, da ein schneller Abfall desBlutzuckers die entscheidende Voraussetzung für das Auftreten eines Insulinschocks ist.

Schließlich möchten wir nicht unerwähnt lassen, daß wir ein weiteres, von Schmid und Arens [6] isoliertes Peptid aus Rinderinsulin geprüt haben soweit es die geringe, zur Verfügung stehende Menge erlaubte. Es handelt sich um die ,Fraktion $b_{2}{ }^{6}[6]$, bei der die vorher kontinuierliche Aminosäurenkette durch Abspaltung von Lys-Arg an der A-Kette unterbrochen und zweikettig wurde. An nüchternen Ratten senkte dieses Peptid den Blutzucker eine halbe Stunde nach subcutaner Injektion von $10 \mathrm{bzw} .25 \mathrm{nmol} / \mathrm{kg}$ signifikant um $12 \mathrm{bzw}$. 22 Prozent der Kontrollwerte.

\section{Literatur}

1. Chance, R.E., and R.M. EluIs : Isolation and characterization of porcine proinsulin. Fed. Proc. 27, 392(1968).

2. Cox, W., E.D. HenLey, and R.H. Williams : Sulfonylureas and diabetes mellitus. II. Preliminary studies of the mechanism of action. Diabetes 5, 366-371 (1956).

3. HARRIS, J.I., and C.H. Lr: The biological activity of enzymatic digests of insulin. J. Amer. chem. Soc. 74, $2945-2946$ (1952).

4. Hoffman, W.S.: A rapid photoelectric method for the determination of glucose in blood and urine. J. biol. Chem. 120, 51-55 (1937).

5. Rubenstein, A.H., S. Cho, and D.F. Steiner: Evidence for proinsulin in human urine and serum. Lancet 1968 I, $1353-1355$.

6. Schmidt, D.D., und A. ARens: Proinsulin vom Rind. Isolierung, Eigenschaften und seine Aktivierung durch Trypsin. Hoppe-Seylers Z. physiol. Chem. 349, 11571168 (1968).

7. Steiner, D.F.: Evidence for a precursor in the biosynthesis of insulin. Trans. N.Y. Acad. Sci. 30, 60-68 (1967).

8. - and P.E. OYER: The biosynthesis of insulin and a probaible precursor of insulin by a human islet cell adenoma. Proc. nat. Acad. Sci. (Wash.) 57, 473-480 (1967).

9. -D. Cunningham, L. Spigelman, and B. Aten: Insulin biosynthesis : Evidence for a precursor. Science 157, $697-700$ (1967).

Dr. W. Puls und Prof. Dr. G. Kroneberg Institut für Pharmakologie der Farbenfabriken Bayer AG, 56 Wuppertal-Elberfeld Friedrich-Ebert-Straße 217 